\title{
Challenge! -
}

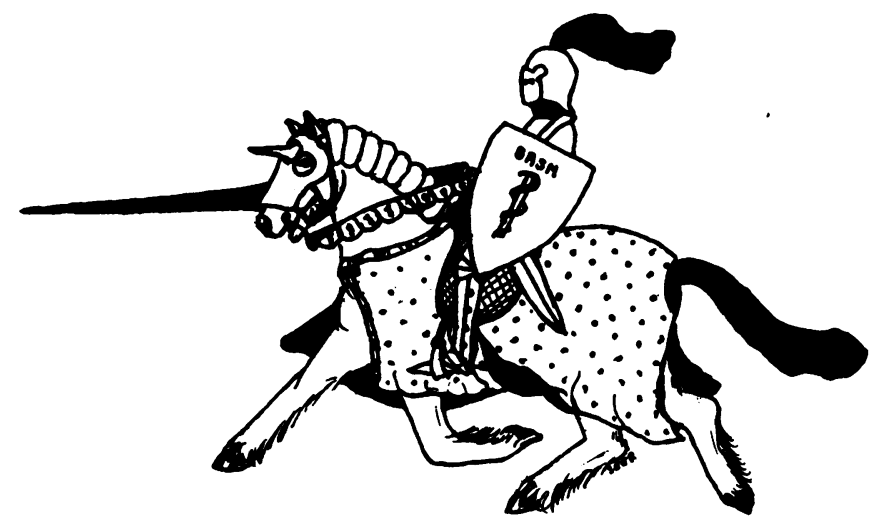

I write to express some reservations about the paper on heel and Achilles pain by Maclellan and Vyvyan (BJSM 1981, 15: 117-121).

The most fundamental fault with this paper is that it lumps all heel pain and all Achilles pain into the same categories. What is "Achilles tendonitis"? No paper is scientifically plausible which appears to claim equally and totally satisfactory outcomes for all Achilles pain from one week's duration in a 17 year old to five years in a 37 year old. There are published differential diagnostic systems for the Achilles tendon in standard texts and I would think a serious paper would take some note of the widely differing pathology present.

Secondly, I don't understand the figures presented. No indication is given of the differing heights of the outcome measures - was there incomplete follow-up, or did some patients die, or what?

Thirdly, the section "Shoes Used" gives little useful information - in most cases brand names only were given. Some of these are incorrect, which may be a matter of sub-editing, for instance is there a make of shoe called "R.O.M." or do the authors mean the Adidas Rom model? This part of the paper is equivalent to saying that people were driving, e.g. a Chrysler or a Leyland, at the time of a road crash.

Previous treatment denoted is unclear. What is meant by "physiotherapy"? Is all physiotherapy the same? If there is controversy about some modalities in respect of Achilles pain, would it not be relevant at least to separate those subjects previously treated by physical contact methods of treatment, e.g. friction, massage, from those untouched?

Next, most people's experience might be that a boy of 17 with a week's history of a sore Achilles tendon would settle normally with a few days of simple rest, plus or minus any specific treatment, or even a heel raise. Is no qualifying period required for a scientific study which compares such a case with the veteran of ten years' suffering (case GD)?

Next, does a claim made in Table I that six children of fourteen years of age or under were all cured rapidly by one sort of heel pad suggest that Sever's Disease need no longer trouble us? Is it indeed valid to lump these cases together with two other subjects aged 67 and 71 , or even the third one of 38 years of age?

It may be that visco-elastic heel inserts are a valuable advance on other types of polyurethane heel supports. No evidence, however, is given in this paper that it is significantly better than any other form of treatment. Statements such as "Since this study a number of patients have returned to sports without their heel inserts and suffered a return of symptoms. These have been swiftly relieved by once again using the heel inserts" and "This result was the same for a patient who had symptoms for seven years and for patients who had had symptoms for only two months" do not offer scientific support for anything.

The discussion of surgery is totally meaningless without classification by differential diagnosis or the indications for surgery. It is also hard to understand why that common cause of Achilles tendon pain - direct pressure from a shoe back - would be relieved by any sort of heel padding. 
The importance of finding new resilient materials for footwear is so important that we cannot risk one possibly promising new compound falling into disrepute because of such an unscientific and subjectively biased paper. Perhaps a communication might be sought outlining the scientific and biologically favourable characteristics of this particular polymer. As a next step, a critical trial based on sound and acceptable differential diagnosis should be sought in the usual way, comparing the clinical merits of the different materials available. Furthermore, it would seem hazardous to standardise all subjects' symptoms without looking at the specially provocative features of their sports activity. Does, for instance, a squash player have to contend with the same forces in the same way as a marathon road runner? This seems highly relevant to any study of this nature.

This paper, in effect, tells us that headache nearly always gets better with Aspirin.

Yours sincerely,

PETER SPERRYN, MB, BS, MRCP, DPhysMed Consultant in Physical Medicine, Hillingdon Athletes Clinic

Hillingdon Hospital, Uxbridge, Middlesex, England

With regard to Dr. Sperryn's challenge I could answer all his points in detail. Clearly some are valid and some are not. The paper I submitted to the British Journal of Sports Medicine with a view to publication was captioned a "pre-

The paper I submitted to the British Journal of Sports Medicine with a view to publication was captioned a "preliminary report". It was precisely that. At the time of submission of the paper there was no clear evidence as to why efficient energy absorption of this type should relieve the symptoms of Achilles tendonitis so consistently. As regards the pain beneath the heel, we simply believe that by the appropriate combination of efficient energy absorption with efficient pressure redistribution beneath the heel, the great majority of patients with pain as a result of local trauma rather than underlying pathology such as Reiter's disease or ankylosing spondylitis will get better. This is as true for "Sever's disease" and "heel spurs" as for the soft tissue bruising, fat pad shear syndrome, and various other conditions related to direct trauma that have been described. Again the observation I made was that this appeared to be a most efficient and consistently beneficial way of managing these problems. In short, the body is able to repair damage even in the presence of continuing activity provided the appropriate physical conditions exist to allow repair to continue. The balance between breakdown and repair is tilted in favour of the patient.

Dr. Sperryn is aware of a subsequent study with numbers up to forty of Achilles tendonitis presented at the International Conference of Sports Medicine in Utrecht 1981, the details of which were given to a Round Table Conference on Achilles tendonitis at that meeting. Those that have used the heel inserts since this paper was published have found a consistently good result and, in fact, a number of high class athletes have avoided surgical decompression of their 